\title{
Evaluating Health Literacy Environments in Australian Health Services
}

Sarah Neil, Kylie Murphy, Glenda Chapman

Charles Sturt University - School of Community Health, PO Box 789, Albury, New South Wales 2640, Australia

Correspondence: smneil2001@gmail.com

\begin{abstract}
The term 'health literacy' refers to the knowledge and skills used by an individual to make decisions about his or her own health. However, the environment in which health decisions are made is increasingly recognised as a critical component of health literacy. The health literacy environment can help to moderate the typical relationship between low individual health literacy and poor health. Becoming a more health literate healthcare organisation may require only meager financial investment for relatively large effectiveness gains. In this article, a review of Australian government health policies identifies three major foci relevant to the health literacy environment: the complexity of health services, the content of health information, and the physical environment. An overarching theme identified in this review is the importance of consumer input in evaluating all aspects of the health literacy environment.
\end{abstract}

\section{Evaluating Health Literacy Environments in Australian Health Services}

Individual health literacy refers to all the skills involved in finding, understanding and using health information to make decisions about
Despite major policy imperatives and the ongoing need to ensure health investments are socially equitable and cost-effective, there is little published evidence of Australian healthcare services evaluating their own health literacy environment. This article establishes the importance of evaluating the health literacy of Australian healthcare services and reviews four potentially useful evaluation tools.

Keywords: health literacy; environment; health service; evaluation; health policy; equity. one's own health. [1] The health literacy environment draws on the health literacy of consumers by challenging them to interpret varyingly accessible information to access and benefit from healthcare. That environment comprises the healthcare system, 
infrastructure, policies, processes, and employees. [1] Environmental health literacy can seem a relatively drab topic. However, cultivating a health literate environment is critical in enhancing health outcomes, reducing health disparities, and increasing the cost-effectiveness of health expenditure. [1]

The definition of health literacy has developed since it first appeared in scholarly literature. This review explores this conceptual evolution. The importance of the health literacy environment is then argued, based on its potential role in achieving major current Australian healthcare policy goals. It is argued that these goals are not only important reasons to improve Australian health literacy environments, but that they should also inform the way health literacy environments are evaluated. Considering current health policy goals, four potentially useful evaluation tools are briefly appraised.,

\section{The Conceptual Evolution of Health Literacy}

When the term 'health literacy' first appeared in academic literature in 1974, it was in the context of ensuring minimal standards of school-based health education. [2] The concept was expanded by the World Health Organisation (WHO) in 1981 [3] when it formally defined health literacy as '...an elementary understanding of nutritional and health needs and of how to prevent or control common health problems'. This definition remains relevant; while the term has since taken on a broader meaning, including recognition of relevant environmental factors. Although recognised by the WHO in 1981, it was not until the 1990's that health literacy began to regularly appear in scholarly literature. $[3,4]$ There was particularly strong engagement with the construct in the domains of public health, health education, health promotion and primary prevention. The 'public health' model viewed health literacy as an asset which can be influenced by education aimed at empowering patients and the public. [5] As early as 1990, researchers such as Glanz and Rudd called for health information to be tailored for patients with low literacy levels. [6] Later in the 1990's, especially in America, attention shifted to the poor health literacy levels of many patients. [5] This 'clinical' model viewed health literacy as a personal deficit linked to non-compliance with health recommendations. [7] In the decades that followed, the 'public health' and 'clinical' views both continued to attract the attention of health academics.

During the late 1990s and early 2000s, more attention was given to measuring and analyzing the relationship between health literacy and health. [8, 9] In Australia in 2000, Nutbeam published 'Health literacy as a public health goal' [10], heralding a resurgence of interest in health literacy as a public health issue. Shortly after in Washington, Ratzan [2] argued that access to new technology had the potential to advance health literacy, and Curran broadened the health literacy discourse to include verbal and online skills, as well as scientific, media, and cultural knowledge. [8] However, by 2004 , based on the IOM definition of health literacy [11], the concept had extended to refer to health information, health care providers and larger structural systems. Awareness of the health literacy environment was slowly growing.

While there was continued measurement of individual health literacy in the 2000s, a shift was evident towards assessing healthcare providers and health systems in relation to health literacy, rather than only measuring consumer strengths and deficits. [7] A health literate organisation was defined as one which 
actively maximised the accessibility of its health services and information. [4] In 2014, the report of the Victorian Consultation on Health Literacy recommended that the deficit approach, focusing solely on individual health literacy, should be avoided. [12] In recent years, there has been a call for more research to guide healthcare systems, health service managers, and practitioners to improve health literacy environments. [13] This marks a radical shift away from earlier conceptualisations of health literacy.

There is increasing recognition that the healthcare environment is critical in not only enhancing personal health literacy but also in redressing the problematic link between low individual health literacy and poor health outcomes. Policies which respond to this idea will be explored in the next section.

\section{Australian Policy and Guidelines Relating to Health Literacy}

In this section, a number of current Australian and international health policy documents are reviewed in relation to environmental health literacy (see Table 1). These documents include government and non-government publications. Examples of State government policies are taken from NSW and Victoria. Three core themes relevant to environmental health literacy were identified in the recommendations reported in these documents: the complexity of health services, the content of health information, and the physical environment. An overarching theme was also identified: the importance of consumer input and participation.

\section{Complexity of health services}

There is general agreement that the western healthcare system is complex. $[14,15,16]$ It is further agreed that this complexity creates barriers to care and promotes health disparities. $[15,16]$ The complexity confronting consumers includes difficulties in ascertaining which health service is most appropriate for one's needs, ascertaining how to obtain referrals and make appointments, finding out whether treatments are covered by Medicare or private health insurance, and the need to interpret large amounts of often unfamiliar information. [17]

The need to reduce fragmentation and associated barriers to accessing care has been identified as a goal at state, federal and international levels. For example, the Australian Charter of Healthcare Rights states that Australians have a right to accessible healthcare. [18] Addressing barriers to accessing services lies at the heart of the WHO strategy for strengthening health systems. [19] This is also the focus of Criterion 2 of the Australian Council on Healthcare Standards' (ACHS) EQuIPNational guidelines (Standard 11) [20] as well as Priority Areas 3 and 4 of the 4th National Mental Health Plan. [21] The National Aboriginal and Torres Strait Islander Health Plan [22] also calls for action to reduce complexity-related barriers to healthcare. Moreover, the importance of understanding different consumers' challenges in accessing care is also identified in the Men's Health Plan [23] and Women's Health Policy. [24]

\section{Content of healthcare information}

Healthcare providers frequently overestimate the health literacy of the average consumer [16] and fail to ensure meaningful understanding of the information they provide. [15] Health information is often written at a reading level beyond that of most Australian adults. $[1,25]$ Healthcare providers may not identify this mismatch because of 
consumers' unwillingness to admit confusion. $[5,26,15]$ Koh and colleagues [26] and Paasche-Orlow and Wolf [15] note that the stressors often at play when accessing the healthcare system have the potential to erode consumers' health literacy capabilities: capabilities which may be adequate under less stressful circumstances. Consequences of misunderstanding health information can include consumers finding meaning which is inconsistent with the communicator's intention or turning to other resources which are more user-friendly but less reliable. [8]

Table 1: Policy documents reviewed for environmental health literacy themes

Documents published by the

Australian federal government:

State policy documents:

Documents from Australian nongovernment sources:

International document:
Men's Health Plan (Department of Health

and Ageing, 2010)

National Women's Health Policy 2010

(Department of Health and Ageing, 2010)

4th National Mental Health plan (The

Department of Health, 2009)

National Aboriginal and Torres Strait Islander Health Plan 2013 to 2023 (The Department of Health, 2013)

NSW State Health Plan Towards 2021 (NSW Ministry of Health, 2014)

Victorian Health Priorities Framework 20122022 (Department of Health, 2011)

Australian Charter of Healthcare Rights (Australian Commission on Safety and Quality in Health Care, 2008)

National Safety and Quality Health Service Standards (Australian Commission on Safety and Quality in Health Care, 2012)

EQuIPNational Guidelines Standard 11 (The Australian Council on Healthcare Standards, 2012)

EQuIPNational Guidelines Standard 12 (The Australian Council on Healthcare Standards, 2012)

Everybody's Business: Strengthening Health Systems to Improve Health Outcomes: WHO's Framework for Action (World Health Organisation, 2007) 
Australian state and federal policies focus on the importance of providing health information which is meaningful to consumers, tailored for specific groups of consumers, and appropriate for the individual consumer's capacity to understand. For example, the importance of providing healthcare information which is meaningful to consumers and carers was highlighted under Criterion 3 of ACHS' EQuIPNational guidelines (Standard 11) [20] and the National Safety and Quality Health Service Standards [27], specifically the standards regarding medications safety, blood products safety, pressure care, and falls prevention.

Targeting health information to specific groups by using appropriate wording, language, and styles of communication was recommended in criterion 1 of ACHS's EQuIPNational Guidelines (Standard 11) [20], the Women's Health Policy [24], and the Men's Health Plan. [23] The latter two policies highlighted the importance of considering the target consumers' age and stage of life in planning health information which is relevant and meaningful. Similarly, targeting health information to an individual consumer's capacity to understand is covered under Criterion 1 of ACHS's EQulPNational Guidelines (Standard 11) [20], the NSW State Health Plan [28], Goal 3 of the Women's Health Policy [24] and Standard 1 of the National Safety and Quality Health Service Standards. [27]

In general, health information needs to be provided in a language the consumer can understand. [21] This is especially important given the increasing demands on consumers' health literacy regarding interpreting health information, making decisions and articulating preferences. [24] The Australian Charter of Healthcare Rights [18] states that Australians have a 'right to be informed about services, treatment options, and costs in a clear and open way'.

\section{The physical environment}

Another key element of the health literacy environment is the physical environment. One aspect of the physical environment is the wayfinding cues consumers can use to determine where they are, identify where they need to go, and make their way to their destination. Difficulty can arise due to obstacles such as outdated or inaccurate directions, inconspicuous signage, and confusing place names such as "Patient Access Centre". [29] Other design obstacles include circular corridors, nondescript entrances, and obscured signs. [29]

Supporting consumers to find their way independently can reduce costs to a health service. [29, 31] Barriers to wayfinding can lead to stress, anger, anxiety, missed appointments, as well as lost revenue. [30] Identifying elements of the physical environment which impede consumer wayfinding is an important step toward improving the accessibility of health services. This goal was identified in both the Men's Health Plan [23] and National Women's Health Policy. [24] The Department of Health and Ageing also identified the need to improve physical access to healthcare as a key issue in reducing barriers, improving health outcomes, and ensuring equal access to healthcare. $[23,24]$

Another important element of the physical healthcare environment is consumer comfort. Providing healthcare in a comfortable and appropriate environment is recommended in Criterion 1 of ACHS's EQuIPNational Guidelines (Standard 12). [32] The Men's Health Plan [23] recommends a gender-neutral environment including posters and magazines suited to 
males. The Health Research and Educational Trust [33] makes recommendations for improving the healthcare environment in relation to noise, pain management and care, communication, and perceived cleanliness.

Despite the importance which the policy documents place on the physical environment in health literacy, reference to this theme was not found in all reviewed policy documents. This may indicate that the influence of the physical healthcare environment is less widely acknowledged than the other two themes.

\section{Involvement of consumers in development and evaluation}

An important overarching theme identified in the reviewed policy documents was the idea of consumer participation and feedback. This is in line with Declaration IV of the WHO's Alma Ata, that "the people have the right and duty to participate individually and collectively in the planning and implementation of their health care". [34] The Australian Charter of Healthcare Rights also states that Australians have the right to comment on healthcare and have concerns addressed. [18] In general, the evaluation of healthcare provision should be informed by consumer feedback. [27, 28] The National Aboriginal and Torres Strait Islander Health Plan [22] and Standard 2 of the National Safety and Quality Health Service Standards [27] place particular emphasis on continual consumer participation and partnership. It is recommended in the Women's Health Policy [24] and Men's Health Plan [23] that intended audiences should be involved in the production of resources to ensure they are appropriate for the intended consumers.

\section{Assessing the Health Literacy Environment}

Given that environmental health literacy is essential to the provision of effective healthcare, it is imperative that services assess their own health literacy environment including through consumer engagement. [26] Assessment serves to shine a light on the barriers and enablers that may be impacting quality of service. $[13,35]$ Identification of burdensome health literacy demands can be the first step in reducing barriers and providing more accessible and effective care. An important means of assessment is feedback collected from consumers about their experiences and perspectives [1, 12], as noted in much of the policy literature reviewed above.

\section{Existing tools}

Currently, there are four review tools available to guide health services in evaluating their health literacy environment:

The Health Literacy Environment Review [25] is an American resource which includes instructions, checklists, and an action plan, with suggestions for reducing identified barriers. The review activities are designed to be completed by consumers, placing significant emphasis on consumer feedback. This resource assesses print and oral communication as well as the physical environment but only regarding wayfinding. The overall complexity of the service is not assessed.

The Health Literacy Review: A Guide [13] is a resource from New Zealand which includes a step-by-step guide to planning and performing a health literacy review within a health service, together with a guide for developing a health literacy action plan based on the results of the review. Complexity of services is reviewed by asking staff how their organisation practices health literacy, and by two separate interviews with consumers about their experiences. These consumer interviews together with observations of clinical interactions also provide information about the appropriateness of verbal communications. Document analysis is used to assess written resources intended for consumers. The physical environment is also assessed, either by the reviewer or a consumer, with respect to wayfinding.

The Enliven Organisational Health Literacy SelfAssessment Resource [36] is an Australian assessment tool which is based on ten aspirational attributes of a health literate organisation. Each attribute is described, and a 
checklist is provided for the assessor to determine whether the criteria of each attribute are present within the organisation. Notes or plans for future action can be recorded. This resource assesses the physical environment (including accessibility and wayfinding), the content of healthcare information, consumer involvement in service evaluation and development, and elements of complexity of the service, such as how payments are made. A limitation of this review tool is that all findings are based on the opinion of the assessor rather than consumers.

Literacy Audit for Healthcare Settings [37] is an Irish auditing resource which includes a toolkit and best practice guidelines for literacy friendly healthcare settings. The auditing guide covers way-finding, print materials and verbal communication. The range of elements assessed is comprehensive and feedback consists of a checkbox which may be completed by staff, or in some cases, by consumers. The assessor selects from four options which indicate to what extent the element is currently being achieved by the health service. This is useful for developing an overview of organisational health literacy. However, further feedback would be required to isolate specific actions for improvement.

\section{Use of review tools in Australian health services}

Despite the availability of review tools, there are very few published examples of Australian health services taking advantage of health literacy evaluation to improve the quality and safety of their service. Johnson [4] described the assessment of a small rural hospital in South Australia using the First Impressions Activities, which are part of the abovementioned Health Literacy Environment Review. [25] Johnson concluded that health literacy barriers were problematic in the evaluated setting, and recommended the tool for similar services trying to reduce health literacy demands for their consumers.

\section{Conclusion}

This review has described the evolution of health literacy and the health literacy environment. It has also highlighted that environmental health literacy is a focus in Australian and WHO health policies. Evaluating environmental health literacy in Australian healthcare services, and acting to redress identified weaknesses, is essential if Australia's health policy goals are to be met. However, while there is a plethora of research on individual health literacy, there is a dearth of published health literacy environment evaluation in Australian healthcare settings. More published work in this area could help to establish what barriers and enablers exist in Australian healthcare services and guide improvements. The drabness of the term 'health literacy' is unfortunate because the concept is much more important than it is exciting. It is also an area in which meagre investments may return substantial improvements in health outcomes.

\section{Reference}

1.Australian Commission on Safety \& Quality in Healthcare (ACSQHC). Health literacy: Taking action to improve safety and quality. Sydney: ACSQHC; 2014. 2.Ratzan SC. Health literacy: Communication for the public good. Health Promot Int 2001; 16(2): 207-214. $3 . \quad$ World Health Organisation (WHO). Development of indicators for monitoring progress towards health for all by the year 2000. Geneva: World Health Organisation; 1981.

4.Johnson A. First impressions: Towards becoming a health-literate health service. Aust Health Rev 2014; 38(2): 190-193.

5. Kickbusch I. Health literacy: Addressing the health and education divide. Health Promot Int 2001; 16(3): 289297. 
6.Glanz K, Rudd J. Readability and content analysis of print cholesterol education materials. Patient Educ Couns 1990; 16: 109-118.

7.Pleasant A. Health literacy: An opportunity to improve individual, community, and global health. New Directions for Adult and Continuing Education 2011; 130: 43-53.

8.Zarcadoolas C, Pleasant A, Greer DS. Understanding health literacy: An expanded model. Health Promot Int 2005; 20(2): 195-203.

9.Baker DW. The meaning and measure of health literacy. J Gen Intern Med 2006; 21(8): 878-883.

10.Nutbeam D. Health literacy as a public health goal: A challenge for contemporary health education and communication strategies into the 21st century. Health Promot Int, 2000; 15(3): 259-267.

11.Institute of Medicine (IOM). Health literacy: A prescription to end confusion. Washington: Institute of Medicine; 2004.

12.Hill S. Report of the Victorian 2014 consultation on health literacy. Melbourne. Centre for Health Communication and Participation, La Trobe University 2014.

13.Ministry of Health. Health literacy review: A guide. Wellington: Ministry of health; 2015.

14.Kickbusch I. Health literacy: A search for new categories. Health Promot Int 2002; 17(1): 1-2.

15.Paasche-Orlow MK, Wolf MS. The causal pathways linking health literacy to health outcomes. Am J Health Behav 2007; 31(Suppl 1): 819-826.

16.Volandes AE, Paasche-Orlow MK. Health literacy. Am J Bioeth 2007; 7(11): 5-10.

17.Kickbusch I. Health literacy: An essential skill for the twenty-first century. Health Education 2008; 108(2): 101-104.

18.Australian Commission on Safety \& Quality in Health Care (ACSQHC). Australian charter of healthcare rights. Sydney: ACSQHC; 2008.

19.World Health Organisation (WHO). Everybody's business: Strengthening health systems to improve health outcomes: WHO's framework for action. Geneva, Switzerland: WHO; 2007.

20.The Australian Council on Healthcare Standards (ACHS). EQuIPNational guidelines standard 11. Sydney: ACHS; 2012a.

21.The Department of Health. Fourth national mental health plan: An agenda for collaborative government action in mental health 2009-2014. ACT: Commonwealth of Australia; 2009.

22.The Department of Health. National Aboriginal and Torres Strait Islander health plan 2013-2023. ACT: Commonwealth of Australia; 2013.

23.Department of Health and Ageing. National male health policy: Building on the strengths of Australian males. ACT: Commonwealth of Australia; 2010a.

24.Department of Health and Ageing. National women's health policy 2010. ACT: Commonwealth of Australia; 2010b.

25.Rudd RE, Anderson JE. The health literacy environment of hospitals and health centers. Boston: Harvard School of Public Health; 2006.
26.Koh HK, Brach C, Harris LM, Parchman ML. A proposed 'health literate care model' would constitute a systems approach to improving patients' engagement in care. Health Aff 2013; 32(2): 357-367.

27.Australian Commission on Safety \& Quality in Health Care (ACSQHC). National safety and quality health service standards. Sydney: ACSQHC; 2012.

28.NSW Ministry of Health. NSW state health plan: Towards 2021. Sydney: NSW Ministry of Health; 2014 29.Carpman J, Grant M. Wayfinding woes: common obstacles to a successful wayfinding system. Health Facilities Management 2002, 15(2), 22-25.

30.Parnell T. Health literacy in nursing: Providing personcentered care. Secaucus, NJ: Springer; 2014.

31.Sloan Devlin A. Wayfinding in healthcare facilities: Contributions from environmental psychology. Behav Sci 2014; 4(4): 423-436.

32.The Australian council on Healthcare Standards (ACHS). EQuIPNational guidelines standard 12. Sydney: ACHS; 2012b.

33. Health Research and Educational Trust. Improving patient experience through the health care physical environment. Chicago II: Health research and educational trust; 2016.

34. World Health Organisation (WHO). Primary health care: report of the International Conference on Primary Health Care, Alma-Ata. In Unicef (Ed.). USSR: The Organisation; 1978.

35. Rudd RE. The health literacy environment activity packet: First impressions and a walking interview 2010. On-line tools: Health Literacy Studies. Retrieved from http://www.hsph.harvard.edu/healthliteracy/practice/e nvironmental-barriers/

36. Thomacos N, Zazryn T. Enliven organisational health literacy self-assessment resource. Melbourne: Enliven \& School of Primary Health Care, Monash University; 2013. 37.Lynch J. Literacy audit for healthcare settings. Dublin. National Adult Literacy Agency; 2009. 